\title{
INFLUENCIA DE LAACTIVIDAD SOLAR Y DE LA VARIABILIDAD CLIMÁTICA SOBRE LAS GRANDES CRECIDAS EN SUIZA
}

\author{
Juan Carlos Peña Rabadán \\ Servei Meteorològic de Catalunya. Generalitat de Catalunya \\ jpena@meteo.cat \\ Lothar Schulte \\ PaleoRisk Research Group. Departament de Geografia Física i Anàlisi Geogràfica Regional. Universitat de Barcelona \\ schulte@ub.edu
}

\section{RESUMEN}

El artículo presenta la variabilidad de las grandes crecidas en Suiza en el periodo 18002008 a partir de un índice estival construido basado en los daños causados por las avenidas (INU), con el objetivo de analizar la posible influencia de la actividad solar y de la variabilidad climática en las grandes crecidas. La coincidencia de los periodos ricos en avenidas con los observados en otras regiones de regímenes climáticos y fluviales diferentes, hace pensar que son los forzamientos climáticos y los cambios en la circulación general de la atmósfera quienes gobiernan la aparición de estos clústeres temporales de alta frecuencia.

Palabras clave: Inundaciones históricas, Oscilación del Atlántico Norte de verano, actividad solar, variabilidad climática, ${ }^{18} \mathrm{O}$, Suiza.

\section{ABSTRACT}

The paper presents the variability of major floods in Switzerland for the period 1800-2008 from a summer index (INU). The index is constructed from the damage caused by flooding, with the aim of establishing the possible influence of the solar and climate variability on the

Fecha de recepción: julio 2012.

Fecha de aceptación: julio 2013. 
major floods. The coincidence of flood-rich periods with those observed in other regions of different climate and fluvial regimes suggests that climate forcings and changes in the general circulation of the atmosphere are those who govern the appearance of these highfrequency temporal clusters.

Key words: Historical floods, Summer North Atlantic Oscillation, solar activity, climate variability, $\delta^{18} \mathrm{O}$, Switzerland.

\section{INTRODUCCIÓN}

\subsection{Contexto general}

La acumulación de episodios de crecidas en Suiza durante los últimos quince años (Schmocker-Fackel y Naef, 2010a) ha dado un nuevo impulso para retomar este tema a una escala regional y local, poniendo especial énfasis en los aspectos particulares de variables y procesos de alta montaña (Weingartner, 1997; Weingartner et al., 2003; Weingartner y Reist, 2004; Jäggi et al., 2007; Abegg et al., 2007; Burger, 2008; Schulte et al., 2009c; SchmockerFackel y Naef, 2010a y b; Wetter et al., 2011; Wilhelm et al., 2012). La explicación de estos procesos, ponerlos en relación con el clima pasado y su relación con los cambios ambientales, tiene una importancia significativa para el futuro de nuestras sociedades. Sin embargo, las respuestas a la variabilidad climática son muy complejas y pueden variar según la región. En particular, los medios alpinos son climáticamente muy sensibles a los cambios a escala global y a la incidencia de las precipitaciones extremas en el proceso de generación de las inundaciones. Por esta razón, existe una necesidad de llevar a cabo estudios casuísticos que investiguen con la mayor precisión y exactitud el comportamiento y evolución de los sistemas naturales y sociales. En ámbitos de alta montaña, la reconstrucción climática y de los eventos hídricos extremos a resolución decadal o incluso anual de los últimos milenios, se ha realizado generalmente a partir de proxies, tales como registros lacustres, glaciares, dendrocronología y estudios isotópicos de espeleotemas (Chapron et al., 2002; Tinner et al., 2003; Casty et al., 2005; Holzhauser et al., 2005; Boch y Spötl, 2008; Wilhelm et al., 2012). Los Alpes y sus áreas limítrofes disponen también de un número amplio de estudios sobre reconstrucciones históricas de inundaciones y variabilidad climática a partir de fuentes documentales y registros instrumentales (Gees, 1997; Pfister, 1988 y 1999; Luterbacher et al., 2004; Weingartner y Reist, 2004; ALP-IMP, 2006; Burger, 2008; Schmocker-Fackel y Naef, 2010a y b; Wetter et al., 2011).

En el ámbito de los Alpes berneses se han venido desarrollando en la última década estudios centrados en la variabilidad del Holoceno tardío y la dinámica fluvial histórica en las cuencas alpinas (Schulte et al., 2009a; 2009b; 2009c), con el objetivo de proporcionar a las autoridades locales los datos de las grandes crecidas para evaluar los riesgos debidos a las inundaciones. Para ello se consideraron métodos sedimentológicos y geocronológicos aplicados a los registros fluviales de varias secciones de los abanicos deltaicos de la cuenca superior del río Aare, ubicada en el Cantón de Berna. Se analizó la influencia de la variabilidad climática y el uso del suelo en los procesos de agradación y paleo-crecidas. El estudio que aquí se presenta está enmarcado dentro de esta línea de investigación. El fin es evaluar 
las influencias de la variabilidad solar y climática sobre las grandes crecidas en Suiza durante los últimos 200 años, utilizando fuentes documentales, proxies climáticos y registros instrumentales, con el propósito de entender los procesos a escala regional que participan en la aparición de clústeres de alta frecuencia en inundaciones.

\subsection{Definición del problema}

La variabilidad de las grandes crecidas en Suiza (catalogadas como severas, muy severas y catastróficas) para el periodo 1800-2008, se ha determinado a partir de un índice estival (INU) que abarca los meses de julio y agosto, meses caracterizados por acumular los eventos más catastróficos. El índice está construido considerando los daños causados por las avenidas e inundaciones en bienes materiales y pérdidas de vidas humanas, de tal manera que una avenida se ha catalogado como severa si se cuantifican los daños, de acuerdo con la clasificación arbitraria aplicada por Gees (1997) a la base de datos utilizada, entre 2 y 20 millones de francos suizos; se clasifica como muy severa si los daños están entre 20 y 100 millones; y como catastrófica cuando el daño producido por la inundación excede los 100 millones de francos suizos. Remarcar que en la estimación de daños se ha tenido en cuenta la inflación que ha sufrido el franco suizo durante el periodo considerado. En los últimos dos siglos se han identificado tres periodos caracterizados por una alta frecuencia de las avenidas: 1830-1851, 1871-1944 y a partir del año 1972 (Peña et al., submitted 01/03/2013). A grandes rasgos, estos periodos no sólo coinciden con los divulgados en estudios centrados en Suiza (Schmocker-Fackel y Naef, 2010a y b), sino que también son sincrónicos con los publicados para diferentes zonas del continente europeo (Glaser y Stangl, 2004; Benito et al., 2008; Ortega y Garzón, 2009; Glaser et al., 2010). Remarcar que el último periodo, que coincide con la fase climática cálida moderna, muy probablemente es debido a un aumento en la exposición de bienes y personas, en definitiva, por un cambio en la vulnerabilidad del riesgo, más que un cambio en la peligrosidad, es decir, en la variabilidad natural. Este incremento es paralelo al incremento exponencial de los daños económicos observado durante el período comprendido entre 1950 y 2011 (Munich Re Group, 2011), causados por las catástrofes naturales, particularmente en regiones montañosas (Abegg et al., 2007).

La aplicación de un análisis espectral a INU señala periodicidades significativas en 2, 11 y 110 años (Peña et al., submitted 01/03/2013). Este resultado sugiere una conexión con ciclos de escala decadal o centenal relacionados con la variabilidad solar. Schulte et al., 2009b detectan para el flanco norte de los Alpes inundaciones de recurrencia centenal, con una ciclicidad que varía entre los 70 y 150 años, de orden de magnitud igual a la identificada en el análisis espectral aplicado a INU. Esta periodicidad podría estar relacionada con los ciclos solares detectados a escala centenal, como puede ser el ciclo Gleissberg, periodicidad que igualmente ha sido identificada en otros registros sedimentarios (Castagnoli, et al., 1994; Versteegh, 2005). El hallazgo de estas ciclicidades relacionadas con la variabilidad solar, junto a la simultaneidad observada entre los periodos caracterizados por una alta frecuencia en INU y los observados en otras regiones de regímenes climáticos y fluviales diferentes, hace pensar que son los forzamientos climáticos a escala global o hemisférica y los cambios en la circulación general de la atmósfera quienes gobiernan la aparición de estos clústeres temporales de acumulación de grandes avenidas. 
Teniendo en cuenta las consideraciones apuntadas, para evaluar los principales procesos que influyen en la aparición de los periodos con una alta probabilidad en las crecidas más severas e inundaciones en Suiza, se van a utilizar las siguientes variables: dos proxies de escala global o hemisférica, el primero en relación a la variabilidad solar $\left({ }^{10} \mathrm{Be}\right)$ y el segundo en relación a la variabilidad climática $\left(\delta^{18} \mathrm{O}\right)$, junto con una medida directa instrumental (temperatura media anual de Suiza) y un patrón de variabilidad climática de baja frecuencia vinculado al Atlántico Norte (la Oscilación del Atlántico Norte de verano, SNAO). Cuando se utilizan el ${ }^{10} \mathrm{Be}$ y el $\delta^{18} \mathrm{O}$ como proxies de la variabilidad solar y climática, hay que tener en cuenta las observaciones que se detallan a continuación.

El ${ }^{10} \mathrm{Be}$, almacenado en los núcleos de hielo, ha demostrado tener un gran potencial para precisar la actividad solar del pasado (Vonmoos et al., 2006), ofreciendo una oportunidad única para reconstruir la variabilidad solar durante los últimos milenios (Steinhilber et al., 2012). Es importante advertir que la señal de los radionucleidos cosmogénicos contiene también un componente climático introducido por su transporte desde la atmósfera donde se producen hasta el archivo natural donde se almacenan (Vonmoos et al., 2006; Abreu et al., 2012). De esta manera, la concentración de los radionucleidos en una muestra, está integrada por una señal de producción (modulada por la energía solar) y por una señal debida al sistema climático (la mezcla atmosférica, el transporte y la deposición). Por tanto, el ${ }^{10} \mathrm{Be}$ producido en la atmósfera durante un lapso de tiempo determinado es eliminado de la atmosfera en un plazo de 1-2 años a través de las precipitaciones, depositándose en el archivo natural. Este proceso debe tenerse en cuenta en el momento de interpretar la variabilidad solar sobre la base de las concentraciones medidas en los radionucleidos cosmogénicos. Es decir, existe un desfase entre la fecha de producción y la fecha de deposición, que en el caso de ${ }^{10} \mathrm{Be}$ está definido para una escala temporal anual o bianual (Vonmoos et al., 2006; Abreu et al., 2012).

Por su parte, la concentración de isotopos estables de oxígeno en el agua procedente de la precipitación en forma de lluvia o nieve, ha estado considerada como un potente indicador del paleoclima (Stuiver y Grootes, 2000). Se ha observado una estrecha relación con algunos parámetros de relevancia climática como la temperatura del aire en superficie, con unos coeficientes de correlación del orden de 0.5 en escalas temporales de uno a cinco años (Jones et al., 2003). Un punto esencial en la interpretación de la señal temporal generada a partir del análisis del $\delta^{18} \mathrm{O}$ se encuentra en una evaluación adecuada y correcta de si esta señal está vinculada a las fluctuaciones climáticas a nivel local o, si también, refleja las fluctuaciones a una escala regional o, incluso, global. Johnsen et al., 2001 observan grandes similitudes en las variaciones del isotopo durante el último periodo glacial y el Holoceno para seis puntos de observación ubicados en Groenlandia (Camp Century, Dye-3, GRIP, GISP2, Renland y NorthGRIP). Sugieren que las variaciones del isotopo de oxígeno retenidas en los registros de hielo son debidas a un cambio climático a escala regional, respondiendo de una manera suficientemente sensible a las fluctuaciones de temperatura detectadas en otros estudios y otros lugares a lo largo del Holoceno. Para épocas más recientes (tres últimas décadas del s. XX), Rozanski et al., 1992 analizan la variabilidad del $\delta^{18} \mathrm{O}$ para registros procedentes del análisis del agua de lluvia para la Europa Central. Señalan que los cambios a largo plazo del isotopo contenido en la precipitación en regiones ubicadas en latitudes medias y altas están estrechamente relacionados con los cambios observados en la temperatura del aire en superficie durante los últimos treinta años del s. XX. Esta similitud en la variabilidad y en 
las tendencias debe ser debida a que la señal isotópica de la precipitación está controlada por procesos a escala regional, como las condiciones de las regiones fuente de vapor, los patrones de transporte del vapor de agua en la atmosfera, la historia de formación de las masas de aire que comportan la precipitación y la temperatura de la base de la nube.

En definitiva, la utilización de proxies solares y climáticos para evaluar posibles influencias sobre INU, puede generarnos dos tipos de problemas que hay que tener en consideración: en primer lugar, se han identificado desfases temporales entre la producción del ${ }^{10} \mathrm{Be}$ y el $\delta^{18} \mathrm{O}$, y la deposición en los archivos naturales, desfases que pueden variar entre 2 y 40 años (Vonmoos et al., 2006; Abreu et al., 2012); y en segundo lugar, la significación paleoclimática de la señal de ambos proxies a escala regional o global puede crear alguna ambigüedad en su correcta interpretación. A pesar de estas objeciones, las señales generadas en base a ambos proxies son susceptibles de reproducir las fluctuaciones observadas en la variabilidad solar y climática a lo largo de los últimos milenios (Stuiver y Grootes, 2000; Vonmoos et al., 2006). Igualmente habría que señalar que la señal generada a partir del índice INU también es el resultado final de todo un proceso que integra aspectos tanto naturales como antrópicos, no siendo una respuesta directa a un cambio en el comportamiento de algunas de las variables que participan, sino la culminación de toda una serie de causas que la provocan.

\subsection{Objetivos del análisis}

El artículo analiza desde un punto de vista cualitativo y explica de una forma cuantitativa, las influencias de las pulsaciones climáticas de los últimos 200 años, determinadas a partir de la variabilidad solar, las oscilaciones térmicas y los cambios en la circulación general de la atmosfera, sobre los cambios en la frecuencia de las grandes crecidas en Suiza. El análisis, de esta manera, se vertebrará a través de dos líneas principales, que han de evaluar los patrones de comportamiento y definir los clústeres temporales caracterizados por un aumento de avenidas severas y catastróficas:

- El análisis de tipo cualitativo se basa en plasmar de forma gráfica la evolución temporal de INU con el objetivo de detectar los periodos de avenidas severas y ponerlos en relación con los registros de ${ }^{10} \mathrm{Be}$, el $\delta^{18} \mathrm{O}$, la temperatura media anual de Suiza y el SNAO, con el propósito de evaluar si existen patrones de comportamiento comunes.

- El análisis de tipo cuantitativo tiene como finalidad explicar la variabilidad de los distintos parámetros para facilitar el análisis de las influencias sobre INU y, de esta manera, poder definir y explicar los patrones de comportamiento que generan los periodos de alta frecuencia en avenidas e inundaciones en Suiza.

\section{DATOS Y MÉTODOS}

Dada la estructura del estudio, los datos utilizados se organizarán en cuatro grupos diferentes, involucrando a cinco tipos diferentes de registros: los referentes a los procesos hídricos (INU), a la variabilidad solar, determinada a partir de los radionucleidos cosmogénicos $\left({ }^{10} \mathrm{Be}\right)$, a la variabilidad climática, definida en base a las oscilaciones térmicas $\left(\delta^{18} \mathrm{O}\right.$ y temperatura media anual de Suiza), y, por último, aquellos datos a partir de los que se 
calculará el índice de circulación de baja frecuencia para los meses de verano (SNAO). El origen y la descripción de estos datos se detallan a continuación.

- Índice INU para el periodo 1800-2008. Elaboración propia (Peña et al., submmited 01/03/2013). Para la creación de un índice hídrico integrado que explique la variabilidad y la frecuencia de las inundaciones entre los años 1800 y 2008 se han utilizado dos fuentes de datos. La información procedente de la recopilación realizada por Gees (1997) que abarca el periodo comprendido entre 1800 y 1994, y la procedente del Swiss Federal Research Institute WSL (Hilker et al., 2009) para el periodo 19722008. Ambas fuentes de datos están edificadas en base a los daños causados por las crecidas e inundaciones. El índice INU se calcula para la totalidad de Suiza y para los meses de julio y agosto, con el objetivo de evaluar la variabilidad temporal en la frecuencia de las crecidas. El índice se ha creado a partir de una aproximación a la noción de riesgo $(\mathrm{R})$, donde se combinan los conceptos de Peligrosidad (P) y Vulnerabilidad (V), a partir de la relación:

$$
\mathrm{R}=\mathrm{P} * \mathrm{~V}
$$

La variable P se estima a partir de los daños y las pérdidas económicas causadas por las inundaciones. Las categorías de daños se han definido en el apartado 1.2. A cada categoría se le atribuye una magnitud arbitraria (de acuerdo con el límite superior de cada categoría de daños). De esta manera, a las inundaciones clasificadas como severas se les aplica un valor de 20, a las muy severas una magnitud de 50, mientras que a las catastróficas se les asigna un valor de 100. Por lo tanto, cada evento queda definido por un valor de P, que varía entre 20 y 100, indicando la intensidad del fenómeno. Por su parte, la V es definida como una distribución espacial del fenómeno y se calcula teniendo en cuenta los cantones que han estado implicados en cada avenida o inundación, por lo que $\mathrm{V}$ varía entre 1 y 26, que es el número máximo de cantones suizos. Aplicando la ecuación (1) obtenemos un valor de R para cada evento. El índice INU, con una resolución anual, es calculado a partir de la suma de todos los valores de $\mathrm{R}$ correspondientes a los meses de julio y agosto. Finalmente, INU es estandarizado a partir de la media y desviación estándar, ambos parámetros calculados para el período 1800 a 2008.

- Valores medios ${ }^{10}$ Be para el periodo 1800-1982 con una resolución temporal de la serie que varía entre 6 y 10 años. Datos procedentes de Delaygue y Bard (2010): Antarctic Last Millennium ${ }^{10} \mathrm{Be}$ Stack and Solar Irradiance Reconstruction. Se ha procedido a una interpolación lineal de los datos para obtener una serie continua en el periodo considerado.

- Valores medios $\delta^{18} \mathrm{O}$ para el periodo 1800-1987 con una resolución temporal de la serie que varía entre 1 y 4 años. Datos procedentes de Stuiver y Grootes (2000): The GISP2 oxygen isotope record. Al igual que para la serie del ${ }^{10} \mathrm{Be}$, se ha procedido a una interpolación para obtener una serie continua en el periodo considerado.

- Temperatura media anual de Suiza en el periodo 1800-2008. Datos obtenidos del proyecto ALP-IMP (Multi-centennial climate variability in the Alps based on Instru- 
mental data, Model simulations and Proxy data). La temperatura media anual se ha calculado a partir de la media aritmética de los puntos de malla correspondientes a la zona de Suiza (Casty et al., 2005).

- Oscilación del Atlántico Norte de verano, SNAO. Elaboración propia (Peña et al., submitted 01/03/2013) a partir de la principal función empírica ortogonal (EOF), extraída a partir de un PCA en S-Mode. Se ha aplicado a la matriz de covarianza para el periodo 1871-2008 (a resolución diaria) y para el periodo 1800-2008 (a resolución mensual), calculada a partir de las anomalías no estandarizadas de la presión media a nivel del mar de la zona extratropical en la zona del Atlántico Norte y del continente europeo, para los meses de julio y agosto. Se ha computado para el dominio $30^{\circ} \mathrm{N}$ $70^{\circ} \mathrm{N}$ y $30^{\circ} \mathrm{W}-30^{\circ} \mathrm{E}$, con una resolución espacial de $2^{\circ}$ latitud $\mathrm{x} 2^{\circ}$ longitud. Se han utilizado dos fuentes de datos: los proporcionados por el 20th Century Reanalysis V2 a través de la NOAA/OAR/ESRL PSD, Boulder, Colorado (Compo et al., 2011) para el periodo 1871-2008, y los datos de presión en superficie para el Atlántico Norte y la Europa Occidental elaborados por Luterbacher et al., 2002 en el periodo comprendido entre los años 1659 y 1999.

Para dar respuesta al primer objetivo planteado, se analizan las correlaciones que pueden tener algunos forzamientos climáticos y la circulación atmosférica sobre INU en base a los índices y proxies paleoclimáticos, a partir de una representación gráfica integrada de las variables arriba reseñadas.

La evaluación del segundo objetivo se vertebra en un sencillo análisis de las diferentes bases de datos utilizadas, estructurado en las tres siguientes fases:

1. A partir de la muestra total de 209 años definida por INU, se crean ocho categorías que involucran los años que cumplen los siguientes requisitos. Por una parte, dos categorías generales que engloban los años con valores de INU menor o igual a 0 , y aquellos con valores de INU mayor a 0 , es decir, se diferencian los años que ha habido crecida muy severa o catastrófica de los que no ha habido. Por otra parte, los años con crecida se clasifican en 6 categorías diferentes, considerando un INU mayor a 0.5, 1.0, 1.5, 2.0, 2.5 y 3.0 veces la desviación estándar, estimada para el periodo 1800-2008.

2. Se calcula la media aritmética de los valores de ${ }^{10} \mathrm{Be}, \delta^{18} \mathrm{O}$, temperatura media anual de Suiza y de SNAO de los años involucrados en cada una de las ocho categorías previamente definidas. De esta manera, se tendrá un valor objetivo que sintetiza las características de las variables analizadas para todo el espectro de INU, comprendiendo desde los años sin crecidas muy severas o catastróficas, hasta aquellos años con una (o varias) inundaciones catastróficas. En definitiva, se puede analizar el comportamiento de cada una de las variables en relación a INU según la intensidad de la crecida.

3. Por último, y de nuevo para cada una de las categorías, se realiza un conteo de los años en fase positiva/negativa del ${ }^{10} \mathrm{Be}, \delta^{18} \mathrm{O}$, temperatura media anual de Suiza y del SNAO, con el fin de evaluar los resultados en base a un aumento del impacto de INU.

Los valores obtenidos se presentan en forma de tabla para facilitar la comprensión de los resultados en base a cada una de las ocho categorías analizadas. 


\section{RESULTADOS}

\subsection{Análisis cualitativo}

Las relaciones entre el índice INU y las anomalías estandarizadas del ${ }^{10} \mathrm{Be}, \delta^{18} \mathrm{O}$ y el SNAO quedan sintetizadas en la figura 1. Las anomalías se han calculado a partir de la media y la desviación estándar estimadas para la totalidad del periodo considerado por cada una de las variables. Además, se ha aplicado un filtro gaussiano de paso bajo de 11 años, con la finalidad de eliminar las ciclicidades de alta frecuencia, las tendencias y los posibles problemas de autocorrelación. Finalmente, cabe señalar que para la representación gráfica de INU se ha utilizado el umbral de INU>1,5 veces la desviación típica con el objetivo de definir de forma más precisa los periodos ricos en crecidas, diferenciándose estos periodos (señalados en gris claro) de aquellos en los que la frecuencia disminuye e incluso desparece. Remarcar que la escala del $\delta^{18} \mathrm{O}$ está invertida para facilitar su interpretación en relación al resto de índices y proxies.

La señal de INU capta una alta variabilidad temporal, determinada por una alternancia en periodos caracterizados por una alta frecuencia en la presencia de grandes avenidas e inundaciones, y periodos donde la frecuencia es muy baja o incluso inexistente. Se distinguen dos clústeres temporales y dos picos individuales definidos todos ellos por una presencia destacada de las crecidas más severas y catastróficas en Suiza: el primero se extiende entre 1825 y 1855; el segundo entre 1880 y 1935; el tercero correspondería a la inundación registrada en el año 1977; el último pico implicaría las inundaciones que afectaron Suiza los años 2005 y 2007. Cabe señalar que la baja frecuencia en grandes avenidas e inundaciones observada entre 1800 y 1825 también es detectada por Schmocker-Fackel y Naef (2010a) entre los años 1790 y 1810 .

La concentración de ${ }^{10} \mathrm{Be}$ en el hielo depende de la intensidad de la radiación cósmica, además del transporte y la precipitación atmosférica (Vonmoos et al., 2006). Se ha observado una correlación inversa entre la actividad solar y la concentración de ${ }^{10} \mathrm{Be}$, por lo que picos máximos en la concentración del radionucleido corresponderían a fases de baja actividad solar, y viceversa. Este hecho es debido a que los rayos solares dificultan el paso de la radiación cósmica intergaláctica y amortiguan su llegada a la atmósfera terrestre (McCracken et al., 2002). Se han detectado tres periodos de baja actividad solar (señalados en gris oscuro): el primero se alargaría hasta el año 1850, que correspondería a las etapas finales del Mínimo Dalton; el segundo se sitúa entre 1875 y 1920, periodo que corresponde al Mínimo de 1900; y el tercero se extiende entre los años 1970 y 1981. En la figura 1 se puede observar cómo los periodos con una alta frecuencia en avenidas suelen corresponder a periodos caracterizados por un predominio de anomalías positivas de ${ }^{10} \mathrm{Be} y$, por tanto, de baja actividad solar. Este patrón es especialmente marcado durante el Mínimo de 1900. Hay que remarcar que el pico identificado en INU entre 1825 y 1855 se corresponde con un periodo caracterizado por un predominio de anomalías de ${ }^{10} \mathrm{Be}$ positivas, pero sería una fase de transición hacia el máximo solar que se produjo a mediados del siglo XIX. Por último, la inundación registrada en 1977 se relaciona con el retroceso claro de la actividad solar identificado durante los años 70 del siglo pasado.

Se ha utilizado la evolución temporal de las anomalías estandarizadas del $\delta^{18} \mathrm{O}$ como proxy climático, con la finalidad de analizar la variabilidad de la temperatura en los últimos 
doscientos años, en las regiones ubicadas en latitudes medias y altas del hemisferio norte, y con el objetivo de evaluar el comportamiento de INU en relación a este parámetro. A pesar de la variabilidad que se ha observado entre las series temporales, la figura 1 señala que las crecidas más severas tienden a concentrarse en periodos donde predominan las anomalías negativas en la concentración del $\delta^{18} \mathrm{O}$ (resaltadas en gris oscuro), es decir, durante periodos climáticos que pueden calificarse como fríos.

Finalmente, de la figura 1 se puede deducir que por regla general el SNAO está en fase con INU a partir de 1880. Este hecho indica que INU tiende a aumentar en periodos climáticos caracterizados por un predominio de valores positivos de SNAO (señalados en gris oscuro). No parece tan claro en el clúster detectado en INU para el periodo 1825-1855, donde la SNAO tiende a fluctuar alrededor de la media o, incluso, a tener valores negativos. Aparte de estos resultados presentados para el periodo 1800-2008, cabe puntualizar que se ha detectado una fase positiva de la SNAO durante el s. XVIII, concretamente, en el periodo que abarcaría los años 1741 y 1782 (resultados no mostrados, Peña et al., submitted 01/03/2013). Este lapso temporal coincide con un periodo de alta frecuencia de las grandes crecidas en Suiza indicado por Schmocker-Fackel y Naef, 2010a entre los años 1740 y 1780 .

Figura 1

EVOLUCIÓN TEMPORAL DE LAS ANOMALÍAS ESTANDARIZADAS DEL ÍNDICE ESTIVAL INU, EL ${ }^{10} B E$, EL $\delta^{18} \mathrm{O}$ Y EL SNAO PARA EL PERIODO COMPRENDIDO ENTRE LOS AÑOS 1800 Y 2008

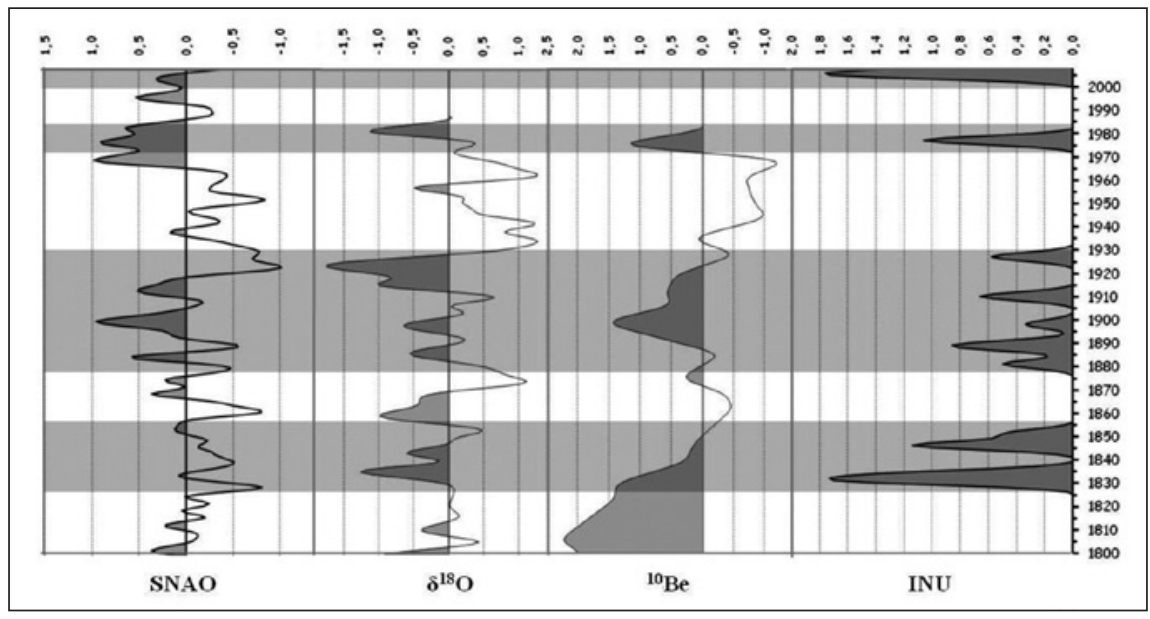

Se ha aplicado un filtro gaussiano de paso bajo de 11 años. Los periodos con una alta frecuencia de crecidas muy severas e inundaciones catastróficas aparecen señalados en el gráfico en color gris claro. Los periodos con anomalías positivas de ${ }^{10} \mathrm{Be}$, anomalías negativas de $\delta^{18} \mathrm{O}$ y anomalías positivas de SNAO están destacados en color gris oscuro.

La figura 2 relaciona las anomalías estandarizadas de INU con las de la temperatura media anual de Suiza para el periodo 1800-2008. A ambas señales se les ha aplicado un filtro gaussiano de paso bajo de 11 años. Se observa que los dos primeros periodos de anomalías positivas de INU están ubicados en la fase fría del periodo analizado que comprende los años 
1825 y 1935. Esta fase fría está en consonancia, si bien con las fluctuaciones propias de la variabilidad climática y solar, con las fases frías reproducidas por el $\delta^{18} \mathrm{O}$ y con periodos de baja actividad solar detectados en la señal del ${ }^{10} \mathrm{Be}$ (Mínimo Dalton y Mínimo de 1900). La inundación datada en el verano de 1977 aparece en un periodo de anomalías de temperatura ligeramente positivas (aunque en un periodo en que se observa un evidente retroceso del $\delta^{18} \mathrm{O}$ y un aumento del ${ }^{10} \mathrm{Be}$ ), mientras que el último clúster de alta frecuencia de crecidas (inundaciones registradas en los años 2005 y 2007), se sitúa en una fase claramente cálida, correspondiente al máximo moderno.

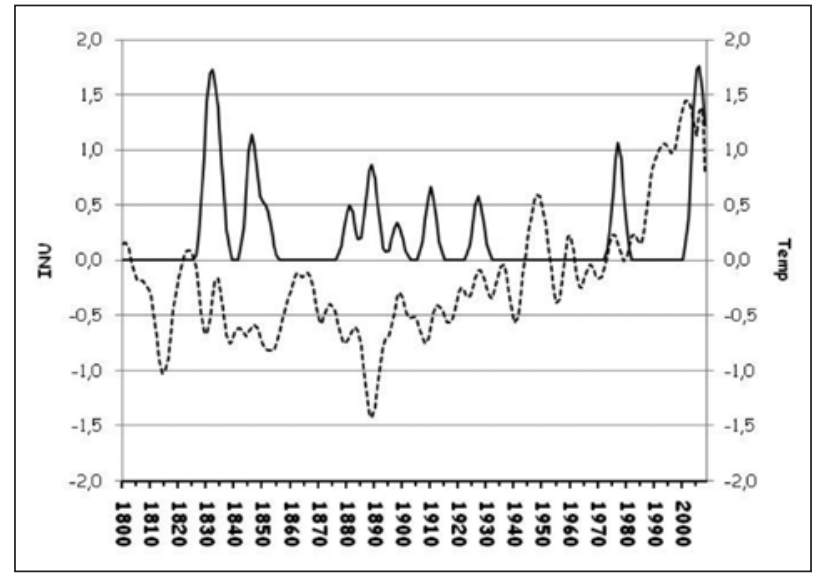

En definitiva, se ha observado un patrón de comportamiento reconocible que relaciona la variabilidad solar y climática con la frecuencia de las avenidas muy severas y catastróficas en territorio suizo. De esta manera, la inundación típica, por regla general, vendría determinada por aparecer en fases de baja actividad solar (valores altos de ${ }^{10} \mathrm{Be}$ ) y climáticamente frías (valores bajos de $\delta^{18} \mathrm{O}$ ), provocada por un episodio de precipitaciones excepcionales (inducidas por patrones sinópticos relacionados al SNAO) y, muy probablemente, precedido por un episodio destacado de temperaturas muy elevadas (aumento del caudal base por una fusión de la nieve o hielo de las cabeceras de las cuencas, Weingartner et al., 2003).

\subsection{Análisis cuantitativo}

La tabla 1 muestra los valores medios de las anomalías de ${ }^{10} \mathrm{Be}, \delta^{18} \mathrm{O}$, temperatura media anual de Suiza en ${ }^{\circ} \mathrm{C}(\mathrm{Tm})$ y SNAO para cada una de las categorías de INU. Se presentan los valores resultantes estructurados en ocho columnas, cada una de las cuales viene definida por una categoría de INU, diferenciando entre ausencia de avenida severa o inundación (INU<0) de las que se ha registrado al menos una avenida severa o inundación (INU>0). Las seis columnas restantes listan los resultados elaborados para cada una de las categorías de INU, 
con un rango que varía de las avenidas de bajo impacto (INU>0.5) a las de más alto impacto (INU>3). Las filas aparecen estructuradas en cuatro bloques correspondientes al ${ }^{10} \mathrm{Be}, \delta^{18} \mathrm{O}$, la temperatura media anual de Suiza (Tm) y la SNAO. Se presenta en la primera fila de cada bloque la media aritmética correspondiente al total de años que quedarían implicados en cada categoría de INU. En las dos filas restantes, se indica el número de años involucrados en la fase positiva o negativa de cada una de las variables analizadas y para cada categoría de INU.

Tabla 1

VALORES MEDIOS DE LAS ANOMALÍAS DE ${ }^{10} \mathrm{BE}, \delta^{18} \mathrm{O}$, TEMPERATURA MEDIAANUAL DE SUIZA EN ${ }^{\circ} \mathrm{C}$ (TM) Y SNAO PARA CADA UNA DE LAS CATEGORIAS DE INU

\begin{tabular}{|l|c|c|c|c|c|c|c|c|}
\hline & INUi<0 & INUi>0 & INUi>0,5 & INUi>1 & INUi>1,5 & INUi>2 & INUi>2,5 & INUi>3 \\
\hline Media ${ }^{10} \mathrm{Be}$ & $\mathbf{0 . 3 2}$ & 0.49 & $\mathbf{0 . 4 6}$ & $\mathbf{0 . 4 6}$ & $\mathbf{0 . 7 0}$ & $\mathbf{0 . 5 2}$ & $\mathbf{0 . 5 2}$ & $\mathbf{0 . 8 5}$ \\
\hline${ }^{10} \mathrm{Be}+$ (años) & 96 & 16 & 10 & 9 & 9 & 6 & 6 & 4 \\
\hline $\mathrm{I}^{10} \mathrm{Be}-$ (años) & 65 & 6 & 4 & 3 & 1 & 1 & 1 & 0 \\
\hline Media $\delta^{18} \mathrm{O}$ & $\mathbf{0 . 0 0}$ & -0.14 & -0.11 & -0.11 & -0.23 & -0.26 & -0.42 & -0.83 \\
\hline$\delta^{18} \mathrm{O}+$ (años) & 87 & 11 & 8 & 7 & 5 & 3 & 1 & 0 \\
\hline$\delta^{18} \mathrm{O}-$ (años) & 79 & 11 & 8 & 7 & 7 & 6 & 6 & 4 \\
\hline Media Tm & 0.00 & -0.02 & 0.02 & -0.04 & 0.02 & 0.08 & 0.31 & 0.95 \\
\hline Tm+ (años) & 91 & 14 & 10 & 8 & 8 & 6 & 6 & 5 \\
\hline Tm- (años) & 93 & 11 & 8 & 8 & 6 & 4 & 2 & 0 \\
\hline Media SNAO & -0.03 & -0.10 & -0.23 & -0.09 & -0.13 & 0.03 & 0.20 & 0.58 \\
\hline SNAO+ (años) & 89 & 13 & 8 & 8 & 7 & 6 & 6 & 5 \\
\hline SNAO- (años) & 95 & 12 & 10 & 8 & 7 & 4 & 2 & 0 \\
\hline
\end{tabular}

Las categorías están definidas en función de un umbral de superación definido a partir de la desviación estándar. Además, se muestran el número de años involucrados en la fase positiva (+) o en la negativa (-) de cada una de las variables. La categoría sin avenida severa aparece diferenciada del resto de categorías. Aparecen señaladas en color gris oscuro y gris claro, respectivamente.

Las medias aritméticas obtenidas de cada una de las variables analizadas, presentan una serie de características que varían en base de la categoría de INU. Los aspectos generales se detallan a continuación.

1. Para todas las categorías, el ${ }^{10} \mathrm{Be}$ presenta valores positivos, incluso para $\mathrm{INU}<0$. Pero es importante señalar que el valor se incrementa a medida que aumenta la categoría de INU, correspondiendo el valor más alto de ${ }^{10} \mathrm{Be}$ a las inundaciones de gran impacto (INU>3). De esta manera, los resultados indican que son los periodos caracterizados por una baja actividad solar cuando se observa una mayor posibilidad de aparición de avenidas e inundaciones de gran impacto.

2. Para todo el espectro de INU, la media del $\delta^{18} \mathrm{O}$ presenta valores negativos, sugiriendo que INU tiende a concentrarse en periodos fríos. Para INU $<0$ ( $\sin$ avenida significativa), el valor sigue siendo negativo pero muy cercano a 0 . En cambio, el valor medio de $\delta^{18} \mathrm{O}$ va disminuyendo a medida que aumenta la categoría de INU, o lo que es lo mismo, aumenta la intensidad de la crecida. Para INU $>3$ el valor medio de $\delta^{18} \mathrm{O}$ es muy negativo (-0.828), revelando que las grandes inundaciones se producen durante las fases climáticas frías.

3. Temperatura media anual de Suiza $(\mathrm{Tm})$ : Si bien para las crecidas que no superan 2 veces la desviación estándar de INU, el valor medio de la anomalía de temperatura 
anual en Suiza se sitúa en torno a $0{ }^{\circ} \mathrm{C}$, es a partir de INU>2.5 cuando las anomalías positivas de temperatura se hacen más grandes. Para la categoría de INU $>3$, es decir, la que alberga las avenidas más catastróficas, la anomalía media anual de temperatura es de $0.95^{\circ} \mathrm{C}$, apuntando que son necesarias pulsaciones cálidas para la aparición de grandes inundaciones.

4. SNAO: Las avenidas más severas en Suiza quedan explicadas por dos patrones de comportamiento. Las avenidas con valores 2 veces inferiores a la desviación estándar están relacionadas a una fase negativa del NAO de verano, mientras que las más severas con valores 2 veces superiores a la desviación tipo se relacionan a una fase positiva, especialmente las catastróficas (INU>3).

Al igual que las medias analizadas de cada uno de los bloques, los años implicados en la fase positiva o negativa de las variables analizadas presentan una variabilidad según la categoría de INU. A continuación se detallan las características más importantes.

1. En general hay un predominio de los años con anomalías positivas de ${ }^{10} \mathrm{Be}$ sobre las negativas, destacando la categoría de mayor impacto (INU>3) donde las cuatro inundaciones que la definen se registraron en años con anomalías por encima de 0 .

2. Para las categorías más bajas (INU<1.5), la distribución de años en fase positiva o negativa de $\delta^{18} \mathrm{O}$ es la misma. Es a partir de INU>1.5 cuando aparecen más años en fase negativa, de forma mucho más evidente a partir de la categoría de INU>2.5. Este resultado indica la preferencia de periodos fríos para las crecidas más catastróficas.

3. Temperatura de Suiza: En todas las categorías hay una mayor acumulación de años en fase positiva (a excepción de la categoría INU>1), pero es especialmente en las más severas cuando la acumulación de años cálidos es más tangible, especialmente en INU>3, cuando las cinco inundaciones registradas en esta categoría ocurrieron en años cálidos.

4. SNAO: La acumulación de años en una fase positiva se hace evidente a partir de INU>2.5, especialmente para las dos categorías de inundación más severa, resultado que evidencia la predilección de los eventos más extremos por este modo de baja frecuencia de circulación atmosférica general a gran escala.

Tal como se había detectado en el análisis cualitativo, se han observado patrones de comportamiento reconocibles que relacionan la variabilidad solar y climática con la frecuencia de las grandes avenidas en territorio suizo, que, de esta manera, refuerza los aspectos detallados en el apartado anterior. De todas formas, hay que remarcar que existen diferencias destacadas entre las avenidas e inundaciones de gran impacto, definidas para un valor de INU superior a 2.5 veces la desviación estándar, de las de impacto inferior, que involucran las categorías inferiores al umbral especificado.

\section{DISCUSIÓN}

El patrón temporal del índice INU, definido por una distribución de clústeres de alta frecuencia no homogéneos en el tiempo y unos patrones temporales coincidentes con otras 
zonas del continente europeo (Glaser et al., 2010), se ha relacionado con una serie de variables con el fin de evaluar y explicar su influencia en la aparición de estos periodos de acumulación de grandes crecidas e inundaciones: la actividad solar $\left({ }^{10} \mathrm{Be}\right)$, las oscilaciones en la temperatura $\left(\delta^{18} \mathrm{O}\right.$ y temperatura media anual de Suiza) y la variabilidad en la circulación atmosférica (a partir del principal modo de variabilidad durante los meses de julio y agosto, el SNAO).

De los resultados obtenidos se puede inferir que las grandes avenidas e inundaciones en Suiza vienen determinadas por aparecer en pulsaciones climáticas frías de baja frecuencia con acumulación de nieve y/o hielo en las cabeceras, provocada por un episodio de precipitaciones excepcionales en combinación con un episodio de calor importante (entre unos días y varios meses de duración, que provoca la fusión de la nieve y/o hielo en cabeceras). A continuación se discutirán detalladamente los procesos implicados.

$\mathrm{El}$ análisis del ${ }^{10} \mathrm{Be}$ indica anomalías positivas del radionucleido en todas las categorías de INU, siendo la categoría INU>3 la que presenta unos valores más elevados. El resultado revela que la presencia de clústeres de gran intensidad en la frecuencia de las grandes avenidas e inundaciones en Suiza está relacionada con periodos caracterizados por una baja actividad del sol. De esta manera, los resultados obtenidos parecen estar en concordancia con los obtenidos por Schulte et al. 2009a , 2009b y 2009c del análisis de los registros sedimentarios extraídos de varias secciones de los abanicos deltaicos de la cuenca superior del río Aare (Alpes berneses). Los resultados de los análisis sugieren una relación entre el calendario de eventos de grandes inundaciones registradas en el flanco norte de los Alpes en los últimos 3600 años y las anomalías positivas de radiocarbono, insinuando un aumento de la frecuencia de las grandes avenidas en periodos de baja actividad solar y, por tanto, durante periodos climáticos caracterizados por unas anomalías térmicas por debajo de los valores normales (Versteegh, 2005). Schulte et al., 2009b proponen la influencia solar como uno de los factores principales desencadenantes de estos eventos hídricos extremos.

El comportamiento del $\delta^{18} \mathrm{O}$ con respecto a INU muestra valores negativos en todas las categorías analizadas, si bien es importante señalar que el valor es más negativo cuanto más severa es la categoría. Además, todos los años en que se ha registrado una inundación implicada en la categoría INU>3 (grandes inundaciones) tienen un valor medio de $\delta^{18} \mathrm{O}$ significativamente negativo. Los resultados indican que las crecidas en Suiza tienden a concentrarse en periodos climáticos fríos, tal como también se ha indicado en otros estudios parecidos. El estado de la cuestión parece consensuar que las variaciones más significativas en la frecuencia de las avenidas aparecen durante las pulsaciones frías (Knox, 2000; Schulte et al., 2009b), especialmente en sus estadios iniciales y finales (Benito y Thorndycraft, 2006), hecho que también se ha podido constatar en Suiza (Schmocker-Fackel y Naef, 2010a).

A pesar de las consideraciones arriba apuntadas, la relación entre la frecuencia de las avenidas en Suiza y la temperatura anual de Suiza señala que los años tienden a tener una anomalía térmica positiva en las categorías de mayor impacto (INU>2.5). Este resultado es complementario al obtenido del $\delta^{18} \mathrm{O}$, aunque parezca que puedan entrar en contradicción. Cabe destacar que uno de los mecanismos que ayuda a intensificar una crecida en ámbitos alpinos es el aporte de agua adicional procedente de la fusión de la nieve o del hielo de las cabeceras (Weingartner et al., 2003). De esta manera, para la formación de nieve y/o hielo en las cabeceras, se necesita de un periodo frío, proceso que será más efectivo cuanto más largo 
sea el periodo de tiempo bajo unas condiciones frías, hecho que favorecerá que la acumulación sea mayor (definido por las anomalías negativas de $\delta^{18} \mathrm{O}$ ). Dentro de este periodo frío, aparecen episodios cálidos (temperatura que puntualmente puede tener unas anomalías muy positivas) que favorecen la fusión de una parte de la masa de nieve y/o hielo que está acumulada en las cabeceras, normalmente de las partes bajas de los glaciares y la masas de nieve acumuladas durante el invierno, masa de agua en forma líquida que entra de forma directa en el drenaje de la cuenca, aumentando el caudal base de los ríos y torrentes, e incrementando el riesgo hidrológico.

El input al sistema hidrológico que genera la avenida es normalmente en forma de agua proveniente de una precipitación abundante y de gran intensidad. La aparición de estos episodios está en relación a unos patrones sinópticos determinados (Jacobeit et al., 2006). Como se ha indicado, el clima de verano en Europa está sintetizado por la SNAO, la cual tiene una fuerte influencia sobre las principales variables meteorológicas a través de cambios en la posición de la trayectoria de las depresiones, no sólo en el Atlántico Norte (Folland et al., 2009), sino también en otras zonas más meridionales del continente europeo (Bladé et al., 2011). Un valor positivo (negativo) de la SNAO se relaciona con un movimiento hacia el norte (sur) de estas trayectorias, fortaleciéndose en fase positiva sobre Islandia y el Mar de Noruega y debilitándose hacia al sur, comportando un tiempo seco y cálido en el centro y oeste de Europa (Folland et al., 2009). En cambio, se ha observado en el sur del continente europeo un tiempo más frío y húmedo durante estas fases positivas (Bladé et al., 2011).

La disposición general de los principales centros de acción en relación a la SNAO se muestra en la figura 3. La configuración a diversos niveles atmosféricos durante la fase positiva de la SNAO está definida a partir de las anomalías diarias (no estandarizadas) de la presión media en superficie (figura 3a) y la altura del geopotencial a $500 \mathrm{hPa}$ (figura 3b). Los mapas están construidos a partir de las composiciones de los días con una calificación por encima de 3 en la matriz de puntuaciones, derivada de la aplicación de un PCA a las anomalías diarias no estandarizadas de presión media reducida a nivel del mar, para el periodo 1871-2008, con la finalidad de determinar el principal EOF de los meses de verano. El geopotencial a $500 \mathrm{hPa}$ resulta de la composición de los días involucrados en el análisis anterior. El umbral definido (>3) caracteriza a una fase muy positiva del modo de circulación. Se observan anomalías negativas sobre la zona mediterránea, en la que cabría incluir Suiza, quedando claramente delimitada esta zona en el geopotencial de $500 \mathrm{hPa}$. La isolínea de 0 metros (anomalías no estandarizadas) enmarca a gran parte del Mediterráneo y el centro-sur del continente europeo, indicando una zona de bajas presiones en estas regiones. La disposición de los centros de acción favorece la inestabilidad explicando la respuesta de la temperatura y de las precipitaciones.

Las perturbaciones, en relación al principal modo de circulación atmosférico general durante los meses de verano, tienen un origen o se profundizan sobre el Mediterráneo, con un recorrido a lo largo de los Alpes, hecho que favorece el ascenso orográfico a través de la cordillera y la producción de precipitaciones intensas de tipo convectivo e, incluso, de tipo advectivo (o combinación de ambos), es decir, episodios con una duración de varios días. De esta manera, la SNAO resulta tener un control muy importante de la variabilidad climática durante la época estival, no tan sólo sobre en el noroeste de Europa, tal como indicaban Folland et al. (2009), sino también en zonas más meridionales del continente (señalado en el 
análisis de Bladé et al. (2011) sobre los impactos de la SNAO en el Mediterráneo). Esta configuración general justifica, en cierto modo, el control que puede ejercer la SNAO sobre INU, hecho que había sido observado de forma cualitativa en la figura 1: se sugería un aumento en la frecuencia de grandes inundaciones en Suiza durante los periodos cuando las fases positivas de la SNAO son predominantes.

A)

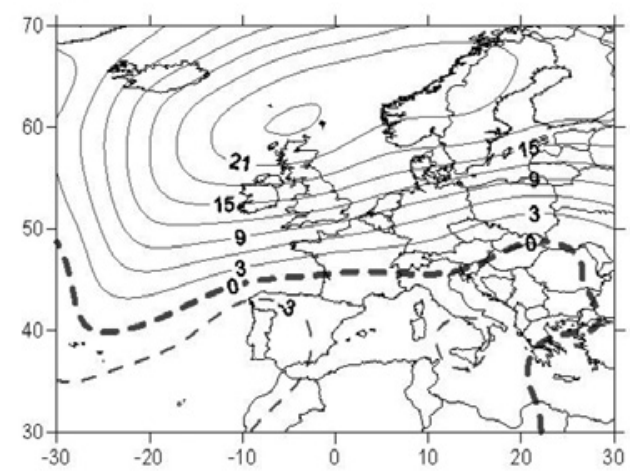

B)

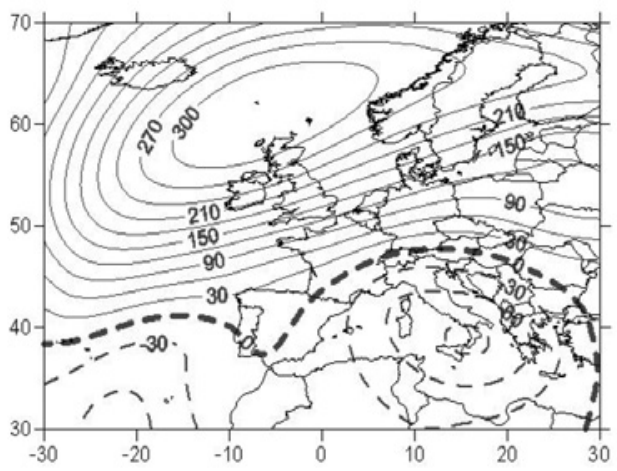

Se ha aplicado a los datos aportados por el proyecto 20th Century Reanalysis de las anomalías diarias no estandarizadas de presión media reducida a nivel del mar, ponderada a partir de la raíz cuadrada de la latitud, para el dominio $30^{\circ} \mathrm{N}-70^{\circ} \mathrm{N} ; 30^{\circ} \mathrm{W}-30^{\circ} \mathrm{E}$ y con una resolución espacial de $2^{\circ}$ latitud x $2^{\circ}$ longitud. El mapa está construido en base a aquellos días con una calificación mayor de 3 obtenida en la matriz de puntuaciones. Unidades expresadas en $\mathrm{hPa}$. Las anomalías positivas aparecen rotuladas en línea continua, mientras que las negativas aparecen en línea discontinua. Se resalta la isolínea de $0 \mathrm{hPa}$. B) Igual que en A) pero para las anomalías de geopotencial a $500 \mathrm{hPa}$. Unidades expresadas en metros.

El análisis de la SNAO en relación a las diferentes categorías de INU, muestra que la explicación arriba desarrollada se puede aplicar a las inundaciones de gran impacto, es decir, para aquellas a partir de un INU>2.5. Las medias aritméticas de la SNAO de los años implicados en estas categorías son claramente positivas. En cambio, las crecidas implicadas en las categorías por debajo de un INU $<2.5$ presentan anomalías negativas, especialmente para INU $<1.5$ y INU $<0.5$, indicando que es la fase negativa del modo de circulación general la que está explicando las crecidas con un menor impacto en el terreno. Esta fase negativa vendría caracterizada por unos patrones sinópticos relacionados con el paso de frentes asociados a bajas centradas entre las Islas Británicas y la Península Escandinava (figura $4 \mathrm{a}$ y $4 \mathrm{~b}$ ). Tal como se había observado en la figura $3 \mathrm{~b}$, la isolínea de 0 metros en la topografía de $500 \mathrm{hPa}$ (figura 4b) divide el centro-norte del continente europeo del centrosur, notando que Suiza queda, a diferencia de lo que se había observado para la SNAO en fase positiva, dentro del primer grupo. De esta manera, la trayectoria principal de las perturbaciones asociadas a este modo de circulación general es del oeste hacia el este, por lo que, a diferencia de la fase positiva, la procedencia de la bajas presiones es atlántica, dando 
un tipo de tiempo más húmedo sobre todo al norte y oeste del continente europeo. Además, las temperaturas menos elevadas provocan que la isolínea de $0{ }^{\circ} \mathrm{C}$ la encontremos en unos niveles atmosféricos más bajos, hecho que determina que la aparición de precipitación en forma de nieve sea mucho más frecuente y, en definitiva, la no transformación de las masas de nieve y/o hielo de los niveles inferiores en escorrentía directa y caudal.

A)

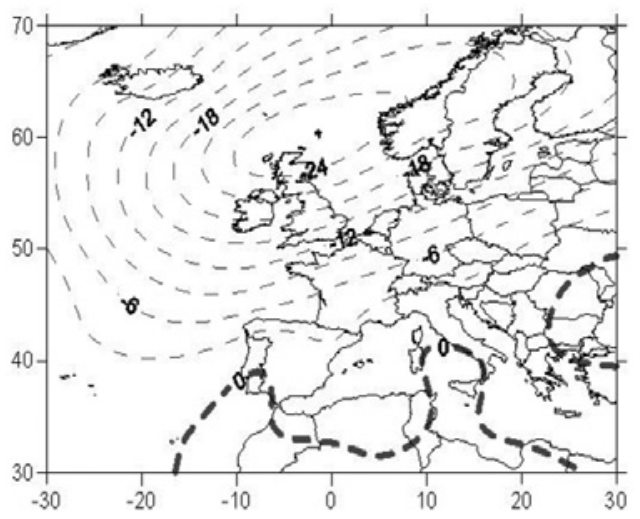

B)

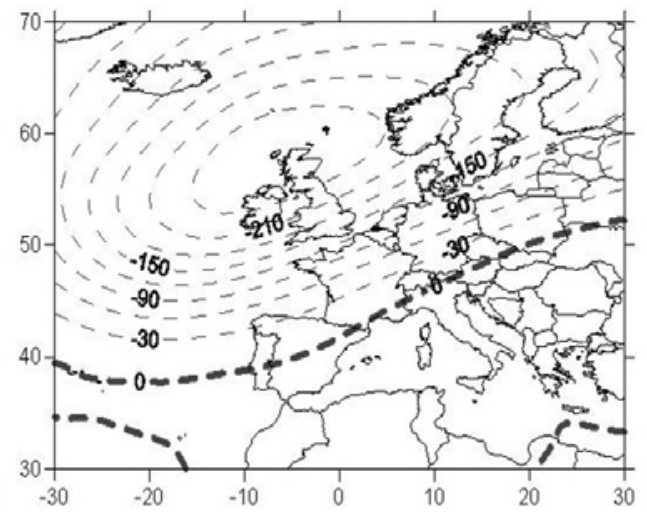

Se han utilizado los datos de las anomalías mensuales no estandarizadas de presión media reducida a nivel del mar aportados por el proyecto 20th Century Reanalysis para el periodo 1871-2008 y por Luterbacher et al., 2002 para el periodo 1800-1999. Se ha conseguido un modo único para el periodo considerado a partir de la regresión del periodo coincidente 1871-1999 de las dos matrices de puntuaciones conseguidas de la aplicación del PCA por separado a cada una de las dos bases de datos utilizadas.

De esta manera, del análisis y discusión de los resultados obtenidos, se pueden deducir dos tipos de comportamientos claramente diferenciados. El primero relacionado con aquellas crecidas de mayor impacto (INU>2.5), mientras que el segundo se relaciona a las crecidas de menor impacto (INU<2.5). Estos dos tipos de comportamiento también se han observado en los resultados obtenidos de los registros sedimentarios extraídos del flanco norte de los Alpes (Schulte et al., 2009c).

- El primer patrón está en relación con las grandes inundaciones en Suiza, caracterizadas por una magnitud de INU mayor a 2.5 veces la desviación estándar (INU>2.5), si bien es en la categoría definida para INU>3, donde los caracteres aparecen mejor definidos. Esta clase involucra a las cinco inundaciones catastróficas que han afectado a Suiza en los últimos 200 años: 1831, 1834, 1846, 1977 y 2005. Estos eventos vienen caracterizados por aparecer en periodos de baja actividad solar (valor medio de ${ }^{10} \mathrm{Be}$ $=+0.85$ ) y climáticamente fríos (valor medio de $\delta^{18} \mathrm{O}=-0.83$ ), fases en que la acumulación de nieve y/o hielo en las cabeceras es más importante, aumentando el riesgo de crecida de forma notable en años cálidos (valor medio de temperatura $=+0.95^{\circ} \mathrm{C}$ ), 
cuando los procesos de fusión son determinantes para la gestación de la avenida. La SNAO está en fase positiva (valor medio de SNAO = +0.56). La trayectoria de las depresiones, normalmente asociadas a ciclones desarrollados sobre el Mediterráneo o el Adriático, tiene una dirección de nordeste (NE) a nord-nordeste (NNE) a lo largo de los Alpes, transportando aire húmedo y comportando lluvias orográficas en las cuencas de montaña. Esta trayectoria es conocida como Vb (van Bebber, 1898; Jacobeit et al., 2006), y produce lluvias extensas e inundaciones catastróficas, especialmente, en la Europa Central (Mudelsee et al., 2004). De esta manera, el resultado está en concordancia con los hallazgos apuntados por Schmocker-Fackel y Naef, 2010b, donde se indica que los periodos de alta frecuencia en avenidas severas en Suiza están en fase con los que se han observado en la República Checa, Italia y España, siendo está dependencia mucho menor cuando se comparan con los periodos ricos en grandes crecidas observados y analizados para Alemania.

- El segundo patrón viene determinado por aquellas crecidas caracterizadas por una magnitud de INU por debajo de 2.5 veces la desviación estándar. Las crecidas están relacionadas a una actividad solar baja y anomalías negativas de $\delta^{18} \mathrm{O}$, pero a diferencia del patrón anterior, las anomalías de temperatura están alrededor de la media y la SNAO muestra valores negativos, especialmente para la categoría de INU $<0.5$, es decir, para la categoría de impacto más bajo (valor medio de SNAO = -0.23). Las crecidas por lo general aparecen en periodos climáticamente fríos pero con unos valores de $\delta^{18} \mathrm{O}$ sensiblemente superiores a los observados en el patrón anterior. Por otra parte, los años relacionados con las crecidas no son cálidos (valor medio = $+0.02^{\circ} \mathrm{C}$ ). Estas consideraciones llevan a pensar que los mecanismos que producen las crecidas son diferentes. La explicación se ha de buscar en los patrones sinópticos relacionados a la fase negativa de la SNAO. Las depresiones en este modo están caracterizadas por frentes fríos de procedencia atlántica, con una trayectoria siguiendo una dirección noroeste a sudeste, canalizados a través de la baja centrada en la latitud de la Península Escandinava y las altas presiones ubicadas en el Océano Atlántico. La configuración es muy parecida a uno de los tipos sinópticos definidos por Jacobeit et al., 2006, implicado en la gestación de grandes crecidas en Europa Central durante los meses de verano (junio a agosto). La persistencia de la situación produce lluvias importantes sobre Suiza y, consecuentemente, avenidas que pueden llegar a tener un impacto considerable sobre el terreno, los bienes materiales y las personas (Barriendos y Rodriguez, 2009).

\section{CONCLUSIONES}

Si bien no parece existir una relación unívoca entre los diferentes procesos que participan en la generación de las grandes avenidas en Suiza, parecen ser los forzamientos climáticos y su influencia en los cambios en la circulación atmosférica los que pueden tener una importancia determinante. Este argumento viene fundamentado por diversos estudios que señalan estas influencias, algunos de los cuales, y entre otros muchos, se detallan a continuación: para la zona de Norteamérica (Knox, 2000); de Centroeuropa (Pfister, 1999; Glaser y Stangl, 2004; Brázdil et al., 2006; Glaser et al., 2010); de la Penín- 
sula Ibérica (Barriendos y Martín-Vide, 1998; Benito et al., 2003; Barriendos y Rodrigo, 2006; Thorndycraft y Benito, 2006); y, finalmente, para la zona de Suiza (Schulte et al., 2009b; Schmocker-Fackel y Naef, 2010b; Wetter et al., 2011; Wilhelm et al., 2012). A pesar de todas estas contribuciones, no existe un consenso general de cómo influyen estos forzamientos sobre este tipo de eventos extremos y, según algunos estudios, no existe un vínculo claro entre los forzamientos solares con los procesos físicos que han intervenido en el clima, tal como se ha indicado en Wanner et al., 2008, en su visión de conjunto sobre el Holoceno. De esta manera, ha de ser de vital importancia investigar más profundamente en la línea de cómo los mecanismos atmosféricos interfieren en la aparición de los clústeres de alta frecuencia en las grandes crecidas e inundaciones, tal como también ha sido sugerido por diversos autores (Knox, 2000; Schmocker-Fackel y Naef, 2010a).

Sin embargo, se trata de un fenómeno complejo, con un gran impacto sobre el terreno, de pocos días de duración, pero con una larga gestación del riesgo hidrológico en base a los meses y a los años anteriores. De los resultados presentados, de carácter todavía preliminar, se puede concluir que Suiza se encuentra en una zona limítrofe entre los distintos modos de circulación que afectan Europa durante la época estival. Dada esta condición, participa de las zonas atmosféricamente inestables que definen la SNAO tanto en su fase positiva como negativa. Por tanto, está influenciada por toda una serie de patrones sinópticos que están relacionados con estos modos de circulación general y que son susceptibles de provocar y generar grandes avenidas e inundaciones. Estos caracteres definen al territorio suizo como un verdadero 'hotspot' en relación a estos eventos hídricos extremos, afectado por una serie de procesos que tienen origen tanto en el área mediterránea (procedencia de la perturbaciones de los golfos de Génova o Venecia), como en la zona atlántica (frentes fríos canalizados entre una baja en la península escandinava y el anticiclón Atlántico).

\section{AGRADECIMIENTOS}

Este trabajo ha sido realizado en el marco de las actividades el Grup de Recerca Reconegut (SGR) PaleoRisk y ha sido financiado por el proyecto de investigación FluvAlps-Plus (CGL2009-06951/BTE) y por el ICREA Academia. La base de datos de inundaciones para el periodo 1972-2008 ha estado proporcionada por el Swiss Federal Research Institute. El conjunto de datos de presión en superficie y geopotencial a $500 \mathrm{hPa}$ han sido facilitados por el Proyecto Twentieth Century Reanálisis a través de la Oficina del Programa Climático del Nacional Oceanic and Atmospheric Administration (NOAA).

\section{BIBLIOGRAFÍA}

ABEGG, B., AGRAWALA, S., CRICK, F. y de MONTFALCON A. (2007): «Climate change impacts and adaptation in winter tourism». En: Climate Change in the European Alps (Agrawala S, Coord.). Paris, France, OECD, 25-60.

ABREU, J.A., BEER, J., STEINHILBER, F., CHRISTL, M. y KUBIK, P.W. (2012): « ${ }^{10}$ Be in Ice Cores and ${ }^{14} \mathrm{C}$ in Tree Rings: Separation of Production and Climate Effects». Space Sci. Rev., doi: 10.1007/s11214-011-9864-y. 
ALP-IMP (2006): Multi-centennial climate variability in the Alps based on Instrumental data, Model simulations and Proxy data. Final report for RTD-project ALP-IMP (EVKCT-2002-00148)

BARRIENDOS, M. y MARTÍN-VIDE, J. (1998): «Secular climatic oscillations as indicated by catastrophic floods in the Spanish Mediterranean Coastal Area (14th-19th centuries)». Climatic Change, $\mathrm{n}^{\circ} 38,473-491$.

BARRIENDOS, M. y RODRIGO, F. S. (2006): «Study of historical flood events of Spanish rivers using documentary data». Hydrol. Sci. J., no 51, 765-783

BARRIENDOS, M. y RODRIGUEZ, R. (2009): Reconstruction of synoptic atmospheric patterns in Western Europe during severe flood events in the Berner Oberland (1780 to 1880). 3rd FluVAlps Meeting, Barcelona, 30.11.2009.

BENITO, G., DÍEZ-HERRERO, A. y DE VILLALTA, M. (2003): «Magnitude and frequency of flooding in the Tagus River (Central Spain) over the last millennium». Climatic Change, $\mathrm{n}^{\circ} 58,171-192$.

BENITO, G. y THORNDYCRAFT, V.R. (2006): «Palaeoflood hydrology: insight into rare events and extreme flood discharges». La Houille Blanche, ${ }^{\circ}$ 5, 91-96.

BENITO, G. THORNDYCRAFT, V.R., RICO, M., SÁNCHEZ-MOYA, Y. y SOPEÑA, A, (2008): «Palaeoflood and floodplain records from Spain: Evidence for long-term climate variability and environmental changes». Geomorphology, nº 101, 68-77.

BLADÉ, I, LIEBMANN, B, FORTUNY, D y VAN OLDENBORGH, G. (2011): «Observed and simulated impacts of the summer NAO in Europe: Implications for projected drying in the Mediterranean region». Climate Dynamics, DOI: 10.1007/s00382-011-1195-x.

BOCH, R. y SPÖTL, C. (2008): «The origin of lamination in stalagmites from Katerloch Cave, Austria: Towards a seasonality proxy». PAGES News, ${ }^{\circ}$ 16, 21-22.

BRÁZDIL, R., KUNDZEWICZ, Z. W. y BENITO, G. (2006): «Historical hydrology for studying flood risk in Europe». Hydrol. Sci. J., nº 51, 739-764.

BURGER, L. (2008): Informationsbeschaffung bei Hochwassersituationen - Dokumentation der grössten überregionalen Hochwasserkatastrophen der letzten 200 Jahre in der Schweiz. Diplomarbeit.

CASTAGNOLI, G. C., BONINO, G. y TARICCO, C. (1994): «Solar magnetic and bolometric cycles recorded in sea sediments». Solar Physics, $n^{\circ} 152,309-312$.

CASTY, C., WANNER, H., LUTERBACHER, J., ESPER, J. y BÖHM, R. (2005): «Temperature and precipitation variability in the European Alps since 1500». Int. J. Climatol, $n^{\circ} 25: 1855-1880$.

CHAPRON, E., ARNAUD, F., A., NOËL, H., REVEL, M., DESMET, M. y PERDEREAU, L. (2002): «Rhone River flood deposits in lake Le Bourget: a proxy for Holocene environmental changes in the NW Alps, France». Boreas, n 34, 404-416.

COMPO, G.P., WHITAKER, J.S., SARDESHMUKH, P.D., MATSUI, N., ALLAN, R.J., YIN, X., GLEASON, B.E., VOSE, R.S., RUTLEDGE, G., BESSEMOULIN, P., BRÖNNIMANN, S., BRUNET, M. ., CROUTHAMEL, R.I., GRANT, A.N. , GROISMAN, P.Y., JONES, P.D., KRUK, M., KRUGER, A.C., MARSHALL, G.J., MAUGERI, M. MOK, H.Y., NORDLI, Ø., ROSS, T.F., TRIGO, R.M., WANG, X.L., WOODRUFF, S.D. y WORLEY, S.J. (2011): «The Twentieth Century Reanalysis Project». Quarterly J. Roy. Meteorol. Soc., n ${ }^{\circ}$ 137, 1-28. 
DELAYGUE, G. y BARD, E. (2010): «An Antarctic view of Beryllium-10 and solar activity for the past millennium». Climate Dynamics, doi: 10.1007/s00382-010-0795-1.

FOLLAND, C.K., KNIGHT, J., LINDERHOLM, H.W., FEREDAY, D., INESON, S. y HURRELL, J.W. (2009): «The summer North Atlantic Oscillation: past, present, and future». J. Climate, $\mathrm{n}^{\circ} 22,1082-1103$.

GEES, A. (1997): Analyse historischer und seltener Hochwasser in der Schweiz. Bedeutung fr das Bemessungshochwasser. Bern (Switzerland) Geographica Bernensia G53.

GLASER, R. y STANGL, H. (2004): «Climate and floods in Central Europe since AD 1000: Data, methods, results and consequences». Surveys in Geophysics, n 25, 485-510.

GLASER, R., RIEMANN, D., SCHÖNBEIN, J., BARRIENDOS, M., BRÁZDIL, R., BERTOLIN, Ch., CAMUFFO, D., DEUTSCH, M., DOBROVOLNÝ, P., van ENGELEN, A., ENZI, S., HALÍČKOVÁ, M., KOENIG, S., KOTYZA, O.., LIMANÓWKA, D., MAKKOVÁ, J., SGHEDONI, M., MARTIN, B. y HIMMELSBACH, I. (2010): «The variability of European floods since AD1500». Climatic Change, nº 101, 235-256.

HOLZHAUSER, H., MAGNY, M. y ZUMBÜHL, H.J. (2005): «Glacier and lake-level variations in west-central Europe over the last 3500 years». The Holocene, no 15, 789-801.

HILKER, N., BADOUX, A. y HEGG, C. (2009): «The Swiss flood and landslide damage database: 1972-2007». Nat. Hazards Earth Syst. Sci., nº 9, 913-925

JACOBEIT, J., PHILIPP, A. y NONNENMACHER, M. (2006): «Atmospheric circulation dynamics linked with prominent discharge events in Central Europe». Hydrological Sciences Journal, $\mathrm{n}^{\circ}$ 51, 946-965.

JÄGGI, M, NAEF, F. y LEHMANN, C. (2007): Hochwasser 2005. Lokale Lösungsorientierte Ereignisanalyse (LLE) Lütschine. Bau-, Verkehrs- und Energiedirektion des Kanton Bern.

JOHNSEN, S. J., DAHL-JENSEN, D., GUNDESTRUP, N., STEFFENSEN, J. P., CLAUSEN, H.B., MILLER, H., MASSON-DELMOTTE, V., SVEINBJÖRNSDOTTIR, A. E. y WHITE, J. (2001): «Oxygen isotope and palaeotemperature records from six Greenland ice-core stations: Camp Century, Dye-3, GRIP, GISP2, Renland and NorthGRIP». Journal of Quaternary Science, $\mathrm{n}^{\circ} 16$ (4), 299-307.

JONES, P.D., MARSH, R., WIGLEY, T.M.L. y PEEL, D.A. (2003): «Decadal timescale links between Antarctic Peninsula ice-core oxygen-18, deuterium and temperature». The Holocene, $\mathrm{n}^{\mathrm{o}}$ 3: 14-26, doi: 10.1177/095968369300300102.

KNOX, J.C. (2000): «Sensitivity of modern and Holocene floods to climate change». Quatern. Science Rev., n 19, 439-457.

LUTERBACHER, J., XOPLAKI, E., DIETRICH, D., RICKLI, R., JACOBEIT, J., BECK, C., GYALISTRAS, D., SCHMUTZ, C. y WANNER, H. (2002): «Reconstruction of sea level pressure fields over the Eastern North Atlantic and Europe back to 1500». Climate Dynamics, $\mathrm{n}^{\mathrm{o}} 18,545-561$.

LUTERBACHER, J., DIETRICH, D., XOPLAKI, E., GROSJEAN, M. y WANNER, H. (2004): «European seasonal and annual temperature variability, trends, and extremes since 1500». Science, $\mathrm{n}^{\circ} 303,1499-1503$.

McCRACKEN, K. G., BEER, J. y McDONALD, F.B. (2002): «A five-year variability in the modulation of the galactic cosmic radiation over epochs of low solar activity». Geophys. Res. Lett., nº 29, doi: 10.1029/2002GL015786. 
MUDELSEE, M., BÖRNGEN, M., TETZLAFF, G., GRÜNEWALD, U. (2004): Extreme floods in central Europe over the past 500 years: Role of cyclone pathway «Zugstrasse Vb». Journal of Geophysical Research, nº109: D23101, doi: 10.1029/2004JD005034.

MUNICH RE GROUP (2011): Annual Report 2011. Publicación online: http://www.munichre.com/publications/302-07342_en.pdf [documento consultado el 10 de Julio de 2012].

ORTEGA, J.A. y GARZÓN, G. (2009): «A contribution to improved flood magnitude estimation in base of palaeoflood record and climatic implications - Guadiana River (Iberian Peninsula)». Nat. Hazards Earth Syst. Sci., nº 9, 229-239.

PEÑA, J.C., SCHULTE, L., BADOUX. A., BARRIENDOS, M. y BARRERA-ESCODA, A. (submitted 01/03/2013): «Control of solar forcing and low-frequency atmospheric circulation patterns on major summer floods in Switzerland». Global and Planetary Change.

PFISTER, Ch. (1988): Klimageschichte der Schweiz 1525-1860. Paul Haupt, Bern.

PFISTER, Ch. (1999): Wetternachhersage: 500 Jahre Klimavariationen und Naturkatastrophen. Paul Haupt, Bern.

ROZANSKI, K., ARAGUÁS-ARAGUÁS, L. y GONFIANTINI, R. (1992): «Relation between long-term trend of Oxigen-18 Isotope composition of precipitation and climate». Science, $\mathrm{n}^{\circ} 258,981-985$.

SCHMOCKER-FACKEL, P. y NAEF, F. (2010a): «Change in flood frequencies in Switzerland since 1500». Hydrol. Earth Syst. Sci, no 14, 1581-1594.

SCHMOCKER-FACKEL, P. y NAEF, F. (2010b): «More frequent flooding? Changes in flood frequency in Switzerland since 1850». Journal of Hydrology, $\mathrm{n}^{\circ} 381,1-8$.

SCHULTE, L., PEÑA, J. C., BURJACHS, F., PERNAS, J., CARVALHO, F., SCHMIDT, Th., BARÓ, M., OLIVA, M., BARRIENDOS, M. y VEIT, H. (2009a): «A 3600-year fan delta record of alpine floods: Potentialities of flood hazard assessment». Geophysical Research Abstracts, $\mathrm{n}^{\circ}$ 11, EGU2009-7611-1, Vienna.

SCHULTE, L., JULIÀ, R., VEIT, H. y CARVALHO, F. (2009b): «Do high resolution fan delta records provide a useful tool for hazard assessment in mountain regions?». International Journal of Climate Change Strategies and Management, $\mathrm{n}^{\mathrm{o}}$ 1, 197-210.

SCHULTE, L., VEIT, H., BURJACHS, F., JULIÀ, R. (2009c). «Lütschine fan delta response to climate variability and land use in the Bernese Alps during the last 2400 years». Geomorphology, $\mathrm{n}^{\mathrm{o}} 108,107-121$.

STEINHILBER, F., ABREU, J.A, BEER, J., BRUNNER, I., CHRISTL, M., FISCHER, H., HEIKKILÄ, U., KUBIK, P.W., MANN, M., McCRACKEN, K. G., MILLER, H., MIYAHARA, H., OERTER, H. y WILHELMS, F. (2012): «9,400 years of cosmic radiation and solar activity from ice cores and tree rings». PNAS, $\mathrm{n}^{\circ}$ 109: 5967-5971.

STUIVER, M y GROOTES, P.M. (2000): «GISP2 Oxygen Isotope Ratios». Quaternary Research, $\mathrm{n}^{\circ}$ 63, 277-284.

TINNER, W., LOTTERB, A.F., AMMANNA, B., CONEDERAC, M., HUBSCHMIDA, P., van LEEUWENA, J.F.N. y WEHRLIA, M. (2003): «Climatic change and contemporaneous land-use phases north and south of the Alps 2300 BC to 800 AD». Quaternary Science Reviews, $\mathrm{n}^{\mathrm{O}} 22,1447-1460$.

THORNDYCRAFT, V.R. y BENITO, G. (2006): «Late Holocene fluvial chronology of Spain: the role of climatic variability and human impact». Catena, $\mathrm{n}^{\circ} 66,34-41$.

van BEBBER, W.J. (1898): Die Wettervorhersage. Stuttgart (Germany). Enke, 2nd Ed. 
VERSTEEGH, G.J.M. (2005): «Solar Forcing of Climate. 2: Evidence from the Past». Space Science Reviews, $\mathrm{n}^{\circ}$ 120, 243-286.

VONMOOS, M., BEER, J. y MUSCHELER, R. (2006): «Large variations in Holocene solar activity: Constraints from ${ }^{10} \mathrm{Be}$ in the Greenland Ice Core Project ice core». J. Geophys. Res., $\mathrm{n}^{\circ}$ 111, A10105, doi: 10.1029/2005JA011500.

WANNER, H., BEER, J., BÜTIKOFER, J., CROWLEY, T.J., CUBASCH, U., FLÜCKIGER, J., GOOSSE, H., GROSJEAN, M., JOOS, F., KAPLAN, J.O., KÜTTEL, M., MÜLLER, S.A., PRENTICE, I.C., SOLOMINA, O., STOCKER, T.F., TARASOV, P., WAGNER, M. y WIDMANN, M. (2008): «Mid- to Late Holocene climate change: an overview». Quaternary Science Reviews, n 27, 1791-1828.

WEINGARTNER, R. (1997): Analyse der räumlichen und zeitlichen Variabilität der Hochwasser in der Schweiz. Schlussbericht zum NFP-31-Projekt, Bern.

WEINGARTNER, R., BARBEN, M. y SPREAFICO, M. (2003): «Floods in mountain areas - an overview based on examples from Switzerland». Journal of Hydrology, no 282, $10-24$.

WEINGARTNER, R. y REIST, T. (2004): «Gotthelfs «Wassernot im Emmental» - Hydrologische Simulation des Extremhochwassers vom 13. August 1837», en Katastrophen und ihre Bewältigung - Perspektiven und Positionen (Pfister, Ch. y Summermatter, S., coord). Bern, Berner Universitätsschriften, Band 49, Haupt-Verlag, 21-41.

WETTER, O., PFISTER, Ch., WEINGARTNER, R., LUTERBACHER, J., REIST, T. y TRÖSCH, J. (2011): «The largest floods in the High Rhine basin since 1268 assessed from documentary and instrumental evidence». Hydrological Sciences Journal, $\mathrm{n}^{\circ}$ 56, 733-758.

WILHELM, B. ARNAUD, F., SABATIER, P., CROUZET, Ch., BRISSET, E., CHAUMILLION, E., DISNAR, J.R., GUITER, F., MALET, E., REYSS, J.L., TACHIKAWA, K., BARD, E. y DELANNOY, J.J. (2012): «1,4 kyrs of flash flood events in the Southern European Alps: implications for extreme precipitation and forcing over the north-western Mediterranean area». Quaternary Research, in press.

Los enlaces a las bases de datos se detallan a continuación:

1) The GISP2 oxygen isotope record (Stuiver and Grootes, 2000), disponible on-line en la página web: http://depts.washington.edu/qil/.

2) Antarctic Last Millennium 10Be Stack and Solar Irradiance Reconstruction (Delaygue y Bard, 2010), disponible en: IGBP PAGES/World Data Center for Paleoclimatology

3) Twentieth Century Reanalysis Project (Compo et al., 2011). Datos disponibles en: http:// www.esrl.noaa.gov/psd/data/gridded/data.20thC_ReanV2.html

4) Reconstruction of Sea Level Pressure fields over the eastern North Atlantic and Europe back to 1500 (Luterbacher et al., 2002):

http://www.ncdc.noaa.gov/paleo/pubs/luterbacher2002/luterbacher2002.html 